Cahiers de recherches médiévales

\title{
Communauté et territoire villageois dans l'ouest de la France
}

$\left(\mathrm{XI}^{\mathrm{e}}-\mathrm{XIII}{ }^{\mathrm{e}}\right.$ siècle $)$

\section{Daniel Pichot}

\section{OpenEdition}

\section{Journals}

Édition électronique

URL : https://journals.openedition.org/crm/1542

DOI : 10.4000/crm.1542

ISSN : 1955-2424

\section{Éditeur}

Honoré Champion

Édition imprimée

Date de publication : 15 mars 2003

Pagination : $9-28$

ISSN : 1272-9752

\section{Référence électronique}

Daniel Pichot, "Communauté et territoire villageois dans l'ouest de la France », Cahiers de recherches médiévales [En ligne], 10 | 2003, mis en ligne le 04 octobre 2007, consulté le 15 décembre 2022. URL : http://journals.openedition.org/crm/1542 ; DOI : https://doi.org/10.4000/crm.1542

Ce document a été généré automatiquement le 15 décembre 2022.

Tous droits réservés 


\section{Communauté et territoire villageois dans l'ouest de la France}

(XI ${ }^{\mathrm{e}}$-XIII ${ }^{\mathrm{e}}$ siècle)

Daniel Pichot

1 Le village médiéval est le fruit d'une complexe alchimie qui voit progressivement se mettre en place une série de liens unissant une communauté humaine. Même si l'on peut constater un début d'évolution pendant le haut Moyen Âge, c'est très largement entre le $\mathrm{X}^{\mathrm{e}}$ et le XIII ${ }^{\mathrm{e}}$ siècle que se dessinent, suivant un schéma nouveau, la trame du peuplement et l'organisation des communautés villageoises, dans le grand Ouest de la France comme souvent ailleurs'. Si la présence d'un bâti organisé, d'un centre religieux, d'une structure sociale est indispensable, la constitution d'un terroir propre et son exploitation ne le sont pas moins ${ }^{2}$. La réunion de ces éléments concourt à définir un village dans les formes que lui connaîtront les siècles suivants. Ce mouvement puissant établit les modalités d'un contrôle de l'espace appelé à perdurer et dont notre monde actuel vit encore largement, c'est dire assez l'intérêt de l'étude.

2 Cependant, la simple évocation du mot fait tout de suite émerger l'image du village groupé bien au centre d'un territoire qu'il domine et sur lequel éventuellement se répartissent quelques modestes écarts, sans parler du cas méridional bien étudié du castrum. Les travaux récents ont montré les nuances qu'il fallait apporter à des schémas trop rigides mais il est légitime de s'interroger sur le cas du village de l'ouest de la France déployé dans le contexte d'un habitat dispersé qui a pu faire douter de sa solidité. Pourtant, un phénomène de regroupement a effectivement pris corps pour constituer des noyaux ecclésiaux, pôles de communautés appuyées sur un territoire.

3 Ce dernier est le fruit d'une élaboration sans doute complexe et malgré quelques textes émanant des puissants, il n'est pas certain que ce soit l'œuvre d'une autorité seigneuriale ou épiscopale ou à tout le moins pas d'elles seules. Le processus de formation du territoire villageois, qui dans l'Ouest semble fortement coöncider avec celui de la paroisse, se présente comme un élément fondamental dont l'examen éclaire la naissance de la communauté. À partir de là, il devient nécessaire de préciser le 
rapport entretenu par les villageois avec cet espace qui leur est propre. C'est alors que nous pourrons, sans doute, comprendre comment une portion d'espace est devenue un véritable territoire c'est-à-dire un espace délimité, approprié. R. Brunet, dans Mondes nouveaux, précise que le territoire « contient une idée d'appropriation, d'appartenance ou, au minimum, d'usage. Il est l'espace dans lequel on vit, dont on vit, que l'on " marque », et pour lequel même on se battrait [...] On parle de territoire en fonction du sentiment d'appropriation d'un espace : il vous appartient et vous lui appartenez »". C'est ce travail sur l'espace par les communautés de l'Ouest qui va retenir notre attention et cela d'autant plus qu'elles passent pour peu structurées dans des régions où $a$ priori il est plus courant d'insister sur l'individualisme.

Délimitation du territoire

4 Malgré les rares textes de fondations nouvelles ou les actes épiscopaux qui en précisent quelques détails, les limites paroissiales ne sont pas toujours fixées avec précision dès les origines. C'est un processus long dans lequel interviennent des catégories très diverses aux intérêts pas toujours convergents qui doivent finir par admettre la prégnance de certains critères. Il nous faut les identifier mais aussi nous poser la question redoutable de la fixité de ces limites dont les communes modernes seraient totalement les héritières. Cela repose trop sur des conceptions administratives modernes pour ne pas faire l'objet d'un réexamen.

5 Sauf pour les paroisses de création récente, il existe un héritage pouvant remonter aux premiers temps de la christianisation, voire avant. Sans accorder une importance excessive au cas de Locronan dans le Finistère, il convient de s'y arrêter. La grande troménie, longue procession qui se déroule sur le pourtour de la paroisse pourrait garder le souvenir de traditions très anciennes, bien antérieures au christianisme et marquerait la pérennité d'un espace vécu ${ }^{5}$. Durant le haut Moyen Âge, notre vision se précise et l'espace tend à s'organiser autour de deux réalités, le vicus et la villa. Cette dernière semble relativement délimitée puisqu'on localise les lieux in villa mais, comme ailleurs, nombre d'indices font douter de son homogénéité et il faut reconnaitre une part de vérité dans l'affirmation d'E. Lorans qui dans le Lochois la voit «très peu vécue comme une unité de vie agraire $»^{6}$. Cependant sa réalité présente une évidente diversité. Si le grand domaine classique avec organisation bipartite n'a guère d'existence, certaines villae s'étendent sur le territoire d'une ou plusieurs communes actuelles. Plus souvent, elles possèdent des dimensions beaucoup plus réduites, très inférieures à une superficie communale et, dans le Perche, G. Louise a justement noté que les villae n'ont légué leur nom qu'à des écarts, pas à des paroisses?. Les vici assez nombreux mais mal connus semblent eux aussi contrôler un espace et dominer fréquemment les villae.

6 Si l'on ne peut parler de réseau paroissial avant le $\mathrm{XI}^{\mathrm{e}}$ siècle, plus de la moitié des églises sont cependant fondées dans ces villae et vici, engendrant progressivement des paroisses ${ }^{8}$. Les grandes villae peuvent alors se perpétuer, l'entité territoriale demeurant sensiblement la même. Grandchamp et Thourie dans les actuels départements de LoireAtlantique et Ille-et-Vilaine, Marcillé, dans la Mayenne en fournissent de bons exemples. Plus au sud, en Anjou, on peut suivre exceptionnellement le cas de Champagné-sur-Sarthe, paroisse avec bourg au $\mathrm{XI}^{\mathrm{e}}$ siècle qui dérive d'une curtis citée au $\mathrm{X}^{\mathrm{e}}$ siècle. De même, nombre de vici se pérennisent ainsi mais beaucoup de villae trop modestes n'ont pas leur lieu de culte et s'intègrent progressivement dans des paroisses 
qui les englobent. Une recomposition de l'espace se fait jour. Près de l'abbaye d'Evron en 987, l'église de Viviers semble associée à une villa comme celle de Vimarcé à un vicus mais celle de Torcé regroupe une curtis du même nom, augmentée de plusieurs masures et d'un domaine ${ }^{10}$.

Dans la partie celtique de la Bretagne, se présente une réalité originale, sans doute héritée du bas-Empire, adoptée et généralisée par les Bretons : la plou. Il s'agit d'une paroisse primitive très vaste, souvent plus de 10000 hectares, qui se définit comme l'espace d'une communauté humaine, religieuse, certes, mais pas seulement ${ }^{11} . A u$ IX ${ }^{\mathrm{e}}$ siècle, la plou d'Anast, future Maure de Bretagne, semble s'étendre dans les mêmes limites qu'aux siècles postérieurs en y incluant les paroisses détachées et déjà en germe dans des chapelles annexes ${ }^{12}$.

Un legs du haut Moyen Âge se décèle donc mais les espaces identifiés à cette époque ne sont pas obligatoirement ceux d'une communauté humaine, la villa n'organise pas totalement la vie des habitants et l'on peut s'interroger sur la structuration de l'espace de la plou. Néanmoins c'est sur ce fonds que s'élabore la réalité de l'époque féodale.

Les deux grands pouvoirs, la seigneurie et l'Église, vont alors exercer une influence incontestable sur l'organisation du territoire en imposant un encadrement poussé des hommes dans le système des villages. Pourtant, cette action n'a peut-être pas la puissance ou du moins les implications que l'on pourrait penser et elle doit tenir compte d'un certain nombre d'éléments

10 La seigneurie entretient des rapports complexes avec le village et ne coïncide qu'assez rarement avec lui. Cela se produit cependant. Comme nous venons de le voir, les domaines du haut Moyen Âge peuvent donner naissance à des seigneuries établies dans un cadre villageois et l'exemple des paroisses castrales est bien connu. Beaucoup de châteaux sièges de seigneurie ont créé des paroisses de dimensions très réduites. En Anjou, les fondations de ce genre par Foulque Nerra et Geoffroy Martel se sont multipliées. Montfaucon et Vihiers, châteaux érigés sur des villae comtales, ont engendré de minuscules paroisses dont le territoire a été prélevé sur une plus $a_{\text {ancienne }}{ }^{13}$. Il faut avouer cependant, que, dans l'ouest, l'avenir de ces créations est souvent urbain et que le modèle n'est pas le plus fréquent ${ }^{14}$.

11 Plus communément, la seigneurie interfère nettement dans la délimitation du territoire des communautés humaines. La paroisse de Rigny, d'origine ancienne, ne fixe que très progressivement ses limites, dans le courant du XII ${ }^{e}$ siècle. Elles épousent alors partiellement, sur leur versant occidental, les contours du parc du château mis en place au XI ${ }^{e}$ ou XII ${ }^{e}$ siècle ${ }^{15}$. Éventuellement, le concours va plus loin. Le territoire de Liré, en Anjou, ne s'est que progressivement étendu et fixé (fig. 1). L'espace mis en valeur se cantonne d'abord sur les pentes de la vallée de la Loire mais le déplacement de la cour seigneuriale sur le plateau au XII ${ }^{e}$ ou XIII ${ }^{e}$ siècle déclenche la constitution de nouvelles tenures qui repoussent les frontières du finage vers leur position actuelle dans le cadre communal tout en les concrétisant par le maintien de bois véraux, système fréquent dans la région ${ }^{16}$. 
Fig. 1. Le finage de Liré (Maine-et-Loire)

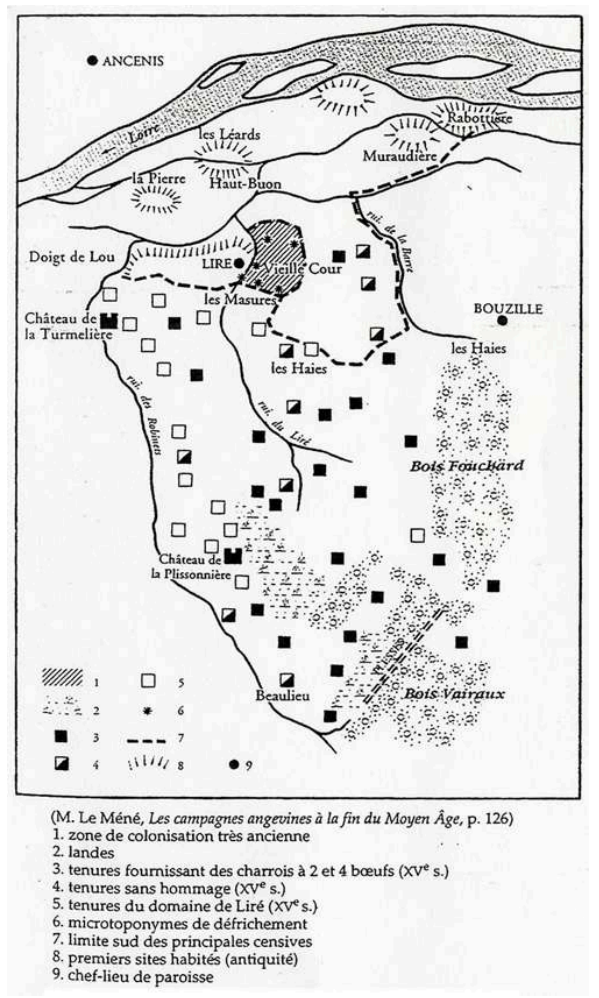

Source : d'après M. Le Méné, Les Campagnes angevines..., in D. Pichot, Le Village éclaté, p. 242.

même ces limites mais il est vraisemblable que, dans certaines situations, il s'agit plus d'une attestation ou d'une corroboration que d'une définition autoritaire. C'est patent lors de la création de paroisses nouvelles en Anjou au XII siècle. Le seigneur de Craon favorise la naissance de la paroisse de La Roë, siège de l'abbaye du même nom fondée par Robert d'Arbrissel. La charte qui relate la cérémonie montre l'évêque demandant à Renaud le Bourguignon de lui proposer un découpage, ce qu'il fait après avoir pris le conseil de ses proches. Certes, il a pu jouer de son pouvoir mais il devait tenir compte de données seigneuriales et paroissiales qu'il a entérinées ${ }^{17}$. Par contre, quand Mathieu, fils de Giraud, demande à l'évêque d'Angers, Ulger, de bénir un cimetière et d'ériger une paroisse sur son domaine récemment défriché de Belle-Noue, la paroisse correspond au domaine et il précise que, jusque-là, ses hommes n'étaient intégrés à aucune paroisse: Viri et feminae terrae meae, quae dicitur Bella Noia, nec in tempore patris mei nec in meo, debuerunt se nec venerunt ulli parrochiae juri parrochiale usquemodo ${ }^{18}$.

Il est d'ailleurs révélateur que les seigneurs voisins élèvent alors des protestations en tant que domini parrochiarum. Ils représentent le groupe important des petits seigneurs de villages dans lesquels ils résident et dont ils portent éventuellement le nom. Cependant, quand la seigneurie coïncide avec le territoire villageois, elle n'en est pas totalement responsable. Bien plus, souvent elle s'y adapte et, dans les grandes seigneuries, la perception des coutumes se calque sur le territoire paroissial pour des raisons d'efficacité, que ce soit pour les cens ou les banalités ${ }^{19}$. Par là même, cependant, l'autorité seigneuriale tend à préciser et renforcer la délimitation d'un territoire. 
14 Néanmoins, c'est le domaine religieux qui joue un rôle majeur, le finage villageois dans l'Ouest tend largement à se confondre avec celui de la paroisse, principale réalité sociale. En voulant encadrer les hommes, l'Église couvre progressivement l'espace d'un maillage complet et inscrit les fidèles dans le cadre précis d'un territoire, ce faisant, elle fonde aussi des communautés conscientes de leurs liens. Quand les églises se sont installées dans des vici ou villae, l'éventuelle continuité des territoires n'a pas signifié obligatoirement celle de l'habitat. À partir du VIII ${ }^{e}$ siècle, les nécropoles et les lieux de culte commencent à se rapprocher et progressivement, avec une nette accélération au cours du XIe siècle, les vivants vont venir s'agréger autour de ce noyau et s'identifier dans une communauté religieuse associant les vivants et les morts dans la continuité des générations. Les lieux de culte, églises paroissiales, chapelles et prieurés ou ermitages présentent alors une certaine diversité pour ne pas dire une certaine anarchie. La réforme grégorienne, en faisant passer les églises paroissiales massivement aux mains des moines, contribue fortement à réaffirmer leur prestige et surtout leur primauté. Autour d'elles, se renforcent et se tissent de nouveaux liens. La paroisse se définit alors dans une série d'obligations et de droits et taxes, prémices, casuel, droits de l'autel parmi lesquelles la sepultura exerce une fonction particulière. L'évêque, s'apprêtant à bénir le cimetière de La Roë, ne demande-t-il pas "quels habitants des forêts d'alentour pourraient être librement paroissiens de ce cimetière ? $»^{20}$. Lieu fort de l'identité paroissiale, ce dernier contribue prioritairement à définir l'espace villageois. Il est la terre consacrée où tous les morts de la communauté, donc d'un espace obligatoirement bien défini, sont appelés à reposer. L'attribution d'un cimetière à un lieu de culte est en général l'étape décisive vers la transformation en église paroissiale et, la réforme pastorale aidant, l'obligation d'y reposer pour tout paroissien sera affirmée. En Bretagne, par deux fois au début du XII e siècle, l'évêque de Saint-Brieuc notifie cette obligation après avoir béni un cimetière et interdit les inhumations ailleurs, en particulier au pied des croix des carrefours ${ }^{21}$.

Les dîmes jouent en ce domaine un rôle moindre. En effet, leur possession se disperse à l'extrême en de multiples mains le plus souvent laïques. Ce n'est que dans le cours du XIII ${ }^{e}$ siècle, et souvent par des politiques volontaristes de rachat, que les institutions ecclésiastiques les font passer sous leur contrôle, les territoires paroissiaux sont alors bien fixés. Néanmoins, la question des dîmes suscite souvent l'intervention des évêques pour des actes de fixation des abornements entre deux paroisses.

De façon, un peu étonnante, dans les quelques actes de création de paroisse que nous conservons, l'Église intervient en priorité mais dans l'énumération des caractères de la fondation, la description des contours, précision attendue en premier, ne vient pas. Quand, Adam, fils d'Urvoy, en 1085, donne la Lande-Huan à Saint-Florent de Saumur pour y installer un bourg de défrichement tenu en coseigneurie, il précise que «Cette terre n'est soumise à aucune paroisse » et s'il envisage l'installation d'une église, il n'est pas sûr qu'elle puisse devenir paroissiale ${ }^{22}$. La clairière prévue n'est pas vraiment décrite et son importance devait être ce que le succès de la fondation allait en faire. Pour l'acte de La Roë, le ruisseau d'Usure et le chemin de Grolet ne déterminent la limite qu'à l'est, il n'est rien dit du reste. Pour Vautorte, l'évêque règle les questions de revenus et de rentes mais ne dit mot des limites pas plus qu'il n'en est précisé lors de la fondation de Brigne $e^{23}$. Cette absence reflète-t-elle le caractère évolutif de fondations pas encore bien installées dans un espace pionnier? Très certainement, mais de façon générale, l'institution ecclésiastique est bien présente dans les opérations de 
délimitation et en ce domaine elle détient quelque initiative mais il n'est pas interdit de penser que ses actes se conforment aussi à des réalités déjà existantes.

L'établissement des limites territoriales ne semble donc pas résulter d'un acte à caractère autoritaire, plus ou moins «administratif » et définitif. Si le Moyen Âge a connu les frontières linéaires, il se réfère aussi souvent au concept de mouvance et la formation du finage villageois résulte de la pratique et d'une histoire du groupe. L'espace de référence varie au cours des âges et le cadre de la villa, sauf exception, n'est pas celui de la paroisse des temps féodaux, en terme de superficie peut-être, mais surtout, de réalité vécue. Si la paroisse de Rigny apparait dans les textes dès le $\mathrm{XI}^{\mathrm{e}}$ siècle, ses limites ne se fixent qu'au cours d'un long processus ${ }^{24}$.

De toute évidence, avant que le contrôle complet du territoire ne soit accompli, l'espace villageois est l'espace vécu et utilisé avec une intensité certainement variable et c'est d'abord cela qui fonde le territoire et l'attachement que lui porte ses habitants. Au $\mathrm{XI}^{\mathrm{e}}$ siècle et même plus tard, les limites précises ne sont pas si courantes, on préfère souvent, et c'est sans doute plus proche de la réalité, dire les limites en désignant le pouvoir voisin. Au tout début du XI ${ }^{\mathrm{e}}$ siècle, quand Alain, duc de Bretagne, confirme le don du monastère de Gahard donné par son vassal Guiddenoch à Marmoutier, il refonde en fait la paroisse et demande aux moines d'accomplir une opération de défrichement, nous sommes donc en zone pionnière malgré l'antiquité du monastère d'ailleurs en ruines. Cet aspect est renforcé par les redevances évoquées : annonaticum, frinscingaticum, fumaticum, vaccaticum, trois sur quatre font référence à l'élevage tandis que la donation insiste fortement sur le pasnage évoquant tant les porcs des moines que ceux des habitants ${ }^{25}$. À la fin, la délimitation encore très certainement imprécise se borne à citer la terra, en fait la zone d'influence des églises voisines qui existent alors : Saint-Aubin d'Aubigné, Saint-Germain de Vieux-Vy, Saint-Martin de Mézières-surCouesnon, dans ce dernier cas est ajoutée cependant la via publica, repère majeur ${ }^{26}$.

Livré-sur-Changeon nous offre un dossier particulièrement intéressant. Vers 1013-1022, Alain III, duc de Bretagne, et son frère Eudes confirment le don de Livré accordé à l'abbaye de Saint-Florent de Saumur par leur père Geoffroy quelque temps auparavant. La charte ne dit rien d'une démarcation mais développe les droits offerts par l'immunitée ${ }^{27}$. Une trentaine d'années plus tard, Conan II confirme de nouveau mais rappelle le tracé défini par Geoffroy : de l'église, l'est est borné par le Vendelais, c'est-àdire la paroisse de Mécé, ensuite le cours de la Veuvre au sud sépare de Dourdain et, à l'ouest, la Brézille, affluent de la Veuvre fait séparation avec le bois de Sévailles. On remonte alors vers le nord pour gagner la route de Rennes par le hameau du Rachat et deux chênes remarquables, le Pouilleux et le Fourchu, ces derniers repères étant sans doute dans Saint-Aubin-du-Cormier de fondation tardive ${ }^{28}$. Il est difficile de dire, d'après cet acte, si le duc se réfère à des limites déjà existantes. En tout cas, il est clair qu'entre les deux paroisses anciennes de Livré et Dourdain, la mise en valeur étant sans doute poussée autour des centres paroissiaux, la frontière est bien matérialisée par les cours d'eau. Vers le nord, en forêt ou sur ses franges, le bornage est bien plus léger. En 1089, le nouveau duc, Alain Fergent se voit sollicité, les anciens de Livré (Livriacenses antiqui) affirment qu'une part de la forêt avait naguère appartenu à la paroisse et à Saint-Florent et demandent en conséquence son rattachement. Un nouveau tracé vient compléter le premier, malheureusement impossible à reconnaître aujourd'hui : « de la haie d'Ingelsende à la Bourgondinière en passant par la croix de Triscan " ${ }^{29}$. La 
concession vaut quand même 10 livres ce qui indique une étendue non négligeable prise sur la foresta du comte. La communauté paysanne de Livré semble bien avoir déjoué une manœuvre ducale. Geoffoy s'était sans doute montré un peu réservé dans sa générosité. Il a voulu conserver une part de sa forêt, bien précieux entre tous, d'ailleurs il se réservait le droit de chasse sur la terre concédée. Cependant, cette zone forestière faisait partie des usages des villageois et c'est cela dont ils imposent le rétablissement. On ne peut donc, même en étant comte, modifier impunément des limites traditionnelles et surtout bien intégrées dans la vie des gens. Au XIII ${ }^{e}$ siècle, une part du territoire sera prélevée par Pierre Mauclerc pour créer Saint-Aubin-du-Cormier mais dans un tout autre contexte. Il n'est donc pas inutile de se demander si les délimitations établies par des autorités seigneuriales ou même par des évêques sollicités expressément n'ont pas tenu compte de ces limites coutumières reconnaissant un lien étroit entre les populations et leur territoire.

20 En conséquence, ces limites ne sont pas si figées que cela. Certes, l'évolution y tend mais une enquête sur quelques paroisses situées autour de la forêt de la Hunaudaye met en lumière des variations au cours des siècles qui ne sont pas toujours négligeables. La stabilité des limites est d'autant plus grande qu'elles s'appuient sur un élément visible et fort: cours d'eau, chemin antique, ce qui est souvent le cas. Pour les paroisses anciennes, il est assez probable qu'elles sont devenues telles quand l'emprise humaine s'est imposée jusque-là, c'est ce qu'invite à penser le cas de Liré. Par contre, celles fondées sur une lisière forestière ou une lande peuvent connaître des changements réels $^{30}$. À Quinténic, les limites liées aux landes se caractérisent par une certaine fluidité. Ce n'est donc pas la limite en soi considérée comme une sorte de frontière administrative qui est conçue mais l'espace qu'elle borne et qui est celui de la communauté. D'ailleurs, très souvent, au moins avant que l'espace entier ne soit bien contrôlé, les espaces périphériques sont peu peuplés, et conservent un caractère de marge, ce qui explique sans doute l'installation des mottes et des domaines qui les accompagnent.

21 L'origine du territoire paroissial est donc complexe, il est le fruit du jeu des autorités, certes, qui peuvent exercer une influence plus ou moins directe mais en tenant compte des pratiques des populations qui, en vivant sur ce territoire et de ce territoire, y puisent une part de leur identité. Il n'est pas surprenant de constater que les réalités villageoise et paroissiale s'affirment en même temps que se précise l'emprise du territoire paroissial. Ce cadre religieux ne paraît donc en rien plaqué et, au contraire, contribue à l'affermissement des communautés ${ }^{31}$. Cela n'implique pas, au moins jusqu'à une organisation complète de l'espace, un caractère quasi "administratif » de ces limites, il s'agit bien d'abord de limites vécues qui fondent une référence sociale et c'est le territoire qui compte plus que les limites, si bien qu'une éventuelle définition en termes linéaires s'accompagne souvent fort bien du caractère très flou de la maitrise des marges. Cette identité spatiale de la communauté surprend un peu dans des zones où l'habitat ne se groupe guère et où l'atomisation de l'habitat semble entraîner celle des groupes.

La communauté et son espace

22 Que le territoire paroissial soit une référence majeure dans la société villageoise s'impose donc comme un fait indéniable, pourtant cela ne va pas sans soulever quelque surprise. Dans un habitat de façon générale dispersé et préférentiellement sous forme de hameaux, quelle est la prégnance de la communauté villageoise et paroissiale ? La 
tradition historiographique insiste plutôt sur la faiblesse des liens communautaires et la force des tendances individualistes. Il ne s'agit pas de vouloir démontrer l'inverse et de manier le paradoxe mais cette vision des sociétés de l'Ouest s'applique sans doute plus à la période contemporaine et sans doute faudrait-il nuancer pour la période féodale. Cependant, on ne peut négliger cette originalité sociale qui détermine un rapport complexe au territoire.

De toute évidence, des liens étroits se tissent entre des groupes restreints et leur territoire, faisant apparemment fi d'une réalité d'un niveau supérieur. La famille est la première des solidarités et elle possède son espace. Le plus souvent, il s'agit d'une tenure qui, devenue très tôt héréditaire, voit les générations se succéder alors que son nom est construit sur celui de la famille, sinon de l'ancêtre fondateur, suivant le schéma plus que fréquent dans l'Ouest, l'adjectif défini féminin précédant un nom d'homme suivi du suffixe « ière/erie » ou " is/aie ». C'est par centaines que l'on compte ces toponymes qui marquent le lien profond du paysan avec sa terre. Au XIII ${ }^{e}$ siècle, l'accord entre les deux demeure solide et la Aillehardière, en Landivy, est occupée vers 1200 par les trois frères Aillehard ${ }^{32}$. Il en va de même pour les noms en La Ville-es ou La Ville-au très répandus dans la région orientale des Côtes d'Armor et tous les noms en ker qui pullulent en Bretagne celtique. Leur formation s'est poursuivie bien après le Moyen Âge mais une bonne part sont nés à l'époque féodale et portent bien le nom d'une terre familiale ${ }^{33}$.

Si l'exploitation souvent modeste, le simple bordage, n'abrite guère qu'une famille conjugale et ses enfants, la situation diffère quand elle présente des dimensions supérieures. La Aillehardière précédemment citée est une masura qui abrite trois frères dont un au moins est marié. L'indivision est alors adoptée, au moins pour un certain temps, en partie par besoin de bras mais aussi par attachement familial au patrimoine héréditaire comme l'affirment bien les trois frères: Hereditarium haberent in predicta masura. Cela pourra donner les parsonniers qui tiennent en frérèches dans les coutumes d'Anjou et Bretagne. Dans cette dernière, elles semblent parfaitement communes au début du XIV ${ }^{\mathrm{e}}$ siècle quand est rédigée la coutume et plusieurs articles y font allusion ${ }^{34}$.

Par extension, le voisinage peut développer des liens de sociabilité particuliers mais l'on atteint pratiquement alors le niveau du hameau, réalité sociale et communautaire de première importance dans tout l'Ouest. Si c'est la forme dominante de l'habitat, il faut reconnaître que le mot recouvre une gamme très ouverte de possibilités. Le plus fréquemment, il s'agit de quelques maisons ou bordages ne regroupant que quelques familles mais on trouve aussi de plus grosses agglomérations qui sont désignées dans la tradition par le terme de "village » traduisant le terme latin villa. Les plus importants peuvent avoir chapelle et même cimetière, si bien qu'ils peuvent devenir eux-mêmes centre paroissial. Ils développent une forte originalité et, bien sûr, assument le contrôle d'un territoire propre. Le hameau tient évidemment dans sa dépendance les terres des bordages et autres exploitations qui le composent. Jardins, ouches champs, prés et landes constituent un véritable finage qui s'individualise clairement dans celui de la paroisse. P. Flatrès l'a d'abord identifié à l'examen des cartes d'état-major. Dans le SudFinistère, certains apparaissent clairement formés d'un bloc cerné par les landes comme quelques exemples relevés dans la presqu'île de Crozon. Les terres cultivées, en dehors du saltus qui n'est pas négligeable, oscillent autour de la centaine d'hectares, chiffre confirmé dans une étude postérieure consacrée à l'ensemble de la Bretagne ${ }^{35}$. Les 
archéologues retrouvent le fait en constatant, par prospection, la diffusion lâche de tessons interprétée comme des traces d'épandage sur des territoires de superficie analogue, entre 50 et 60 hectares, la région de Ruffiac-Carentoir en révèle ainsi plus d'une cinquantaine ${ }^{36}$. Un tel constat suggère d'aller plus loin, l'homogénéité des débris de poterie sur une surface excédant la superficie d'une simple tenure ne peut que témoigner d'une pratique communautaire.

L'hypothèse se renforce à l'étude des parcellaires. Très souvent, les hameaux gardent le souvenir, à leurs alentours, d'un parcellaire laniéré et ouvert, au-delà de la ceinture des jardins et ouches. En Bretagne, depuis longtemps sont connus les méjous, ensembles de champs ouverts mais ceinturés dans leur ensemble par un talus et regroupés près d'un habitat $^{37}$. Le phénomène n'a rien de spécifiquement breton et se retrouve largement dans l'Ouest. J. Renard en étudiant les campagnes de Loire-Atlantique a constaté que des parcelles étroites et non closes caractérisaient les finages de hameaux regroupant des bordages en opposition avec des parcelles beaucoup plus massives se rattachant aux grosses gaigneries ${ }^{38}$. La clairière de Trédéal, dans la forêt de Paimpont, résultant d'un défrichement d'époque féodale comme l'indiquent les micro-toponymes en " erie » et «ais ", sur son finage bien circulaire centré sur un hameau, ne comportait pas de parcelles closes ${ }^{39}$ (fig. 2). Sur les photographies aériennes de la couverture IGN, autour de 1950, alors que le bocage est à son apogée et n'a pas encore connu d'attaque, on ne constate qu'un maigre réseau de haies, d'ailleurs très incomplet. C'était sans doute aussi la situation du bourg de défrichement de la Lande-Huan. De telles formes résultent d'une mise en culture opérée de façon communautaire et rejoignent les hypothèses formulées par le géographe A. Bouhier et systématisées par J. Trochet sur l'organisation d'un paysage ouvert autour de bon nombre de hameaux des régions aujourd'hui bocagères ${ }^{40}$. 
Fig. 2. La clairière de Trédéal (Paimpont, Ille-et-Vilaine)

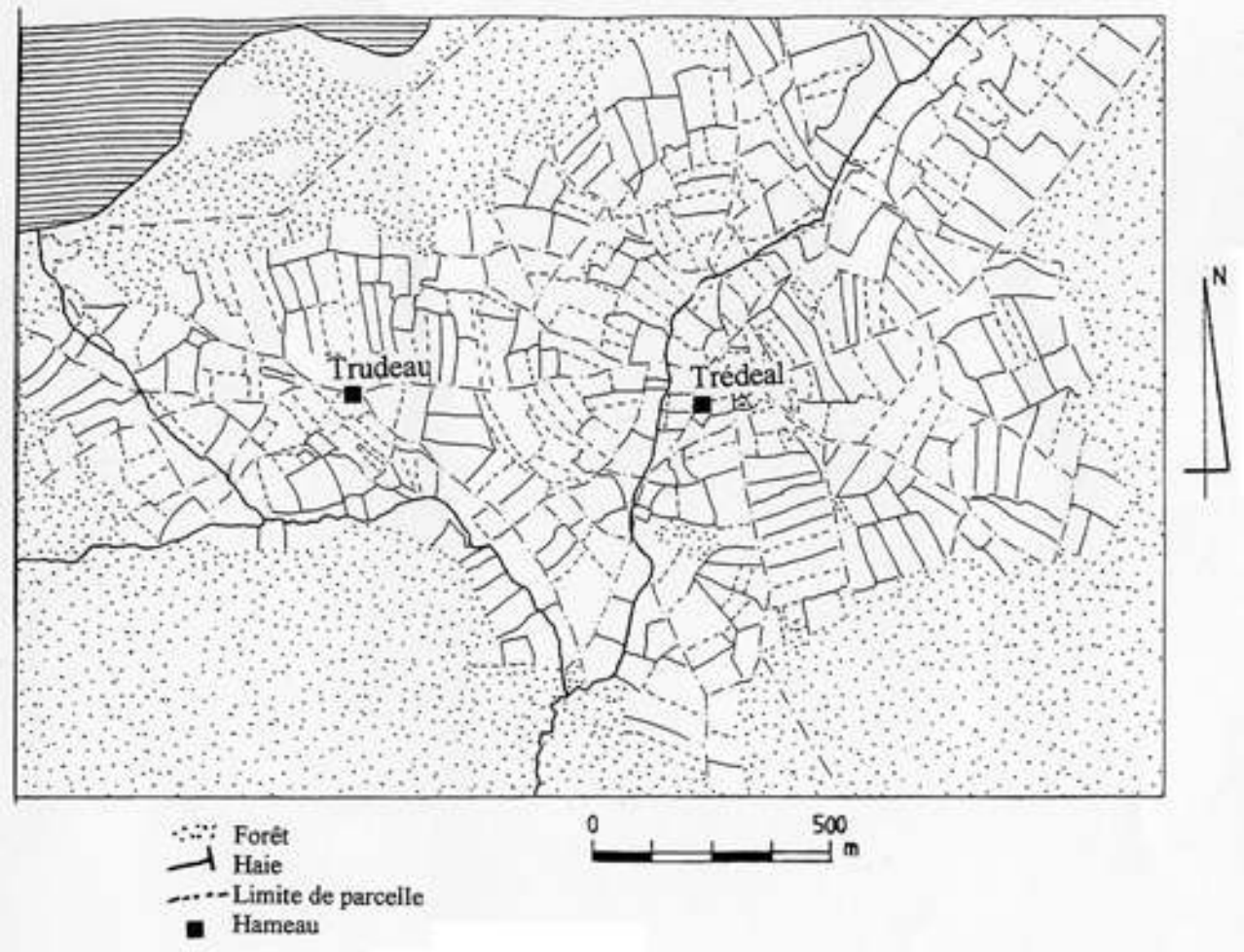

Source : J.-F. Duval, Occupation et mise en valeur du sol dans la région de Plélan-le-Grand au Moyen Âge, Maîtrise, Université Rennes-II, 1998.

Ainsi se trouve mis en évidence un terroir spécifique qui connaît très vraisemblablement une certaine part de travail communautaire, encore plus intense au moment de la mise en valeur. Le gros «village » de Lann-Gouh en Melrand s'organisait autour d'une petite place qui jouait un rôle social et sur laquelle donne le seul four retrouvé pour le moment, tandis qu'à l'arrière d'un groupe de maisons se déploie un vaste espace où des débris de meules indiquent visiblement qu'il était dévolu au travail ${ }^{41}$. On pourrait arguer pour renforcer cette explication de la nature familiale du hameau. Effectivement, quand on peut relever les noms des habitants, il n'est pas rare de retrouver plusieurs membres d'une même famille, pour autant on ne peut parler de hameaux strictement familiaux, sauf peut-être pour de tout petits ${ }^{42}$. Les listes du rentier de la cathédrale de Quimper montrent dans le gros hameau de Lestrefguenc des regroupements limités à quelques unités et surtout de plusieurs familles ${ }^{43}$. On ne peut que suivre $\mathrm{E}$. Reynolds quand elle dénie à ces habitats un strict caractère familial, point n'est besoin des liens du sang pour créer des liens sociaux ${ }^{44}$.

Enfin, ce terroir inclut à peu près obligatoirement des espaces de parcours, des " communs", c'est-à-dire des bois ou landes dans lesquels sont exercés un certain nombre d'usages: élevage, ramassage de bois etc. moyennant des taxes versées au seigneur qui a délivré son autorisation. Très souvent, pour ne pas dire préférentiellement, ce saltus est lié à un hameau beaucoup plus qu'à la communauté paroissiale. Les textes médiévaux confirment les analyses des géographes P. Flatrès et J.R. Trochet qui, s'appuyant plus souvent sur les textes d'Ancien Régime, voire du $\mathrm{XIX}^{\mathrm{e}}$ siècle, ont montré que les terres communes étaient des biens sectionnaux beaucoup plus que des biens paroissiaux ou communaux. Un certain nombre de chartes 
évoquent la communitas, le terme est parlant, dont jouissent un certain nombre de personnes. Il peut s'agir d'une catégorie de dépendants, par exemple ceux d'une abbaye ou, ce qui revient souvent au même, à un hameau ou groupe de hameaux, c'est-à-dire d'une subdivision de la paroisse. Les hommes de la terre de Courbefosse en Fougerolles auront cette communitas pour le ramassage du bois et le droit de pastura ${ }^{45}$.

Un lien communautaire très fort a été parfois mis en lumière et l'exemple des grandes borderies de la forêt du Lattay étudiées par M. Le Mené n'a peut-être pas de caractère exceptionnel. Les religieuses de Notre-Dame du Ronceray d'Angers installèrent, au $\mathrm{XI}^{\mathrm{e}}$ siècle, des pionniers dans des clairières de cette forêt. Plusieurs familles constituèrent un gros hameau, une borderie, qui organisa autour de lui un finage d'une bonne cinquantaine d'hectares grossièrement circulaire et ouvert. Les censiers du $\mathrm{XV}^{\mathrm{e}}$ siècle mettent en évidence l'indivision qui régna dans les premiers temps et sans doute longuement, les familles demeurant collectivement solidaires du paiement des taxes ${ }^{46}$.

La cohésion du groupe peut se renforcer par le biais du domaine religieux. Les gros hameaux sont fréquemment dotés d'une chapelle en Bretagne. Un chapelain assure la desserte du culte et parfois un cimetière est même établi. L'abbaye de Paimpont a installé des prieurés dans certaines clairières de la forêt et les plus dynamiques comme ceux de Trécoët ou du Cannée peuvent, grâce à leur chapelain, devenir des centres de rayonnement et d'attraction. Le cas extrême est atteint avec les trèves. En Bretagne, les plou se maintiennent mais supportent la création de lieux de culte secondaires qui ne sont pas loin d'assumer de vraies fonctions paroissiales. Le terme trevia n'apparait qu'au XIII siècle mais la réalité se dessine auparavant. Vers 1140 , la paroisse de Plouasne possède, outre son église mère, quatre chapelles qui deviendront plus tard centres de paroisses ${ }^{47}$. D'autres conserveront un statut dépendant et certaines paroisses primitives atteignent le chiffre de quatre trèves comme Noyal-Pontivy dans l'actuel département du Morbihan. Dans le diocèse de Quimper, la proportion des trèves bouleverse le système habituel des paroisses.

31 Un hameau ou plusieurs peuvent aussi en Bretagne occidentale définir un territoire reconnu comme tel. La treb puis tref (tribus en latin) désigne un terroir avec ses habitats, en général plusieurs «villages» qui se présente comme un quartier de paroisse. Il est suffisamment défini pour porter un nom qui l'individualise. Vers 1060, Pleubian comporte sept tref ${ }^{8}$. En 1146, une confirmation de Conan III en cite trois: tribus Guynnyn, tribus Guennou, tribus sancti Tuiani, le dernier porte un nom de saint et le premier pourrait être Saint-Guénin, trève de Plourai en Morbihan ${ }^{49}$. Ces deux derniers exemples pourraient orienter vers une explication religieuse que l'on ne peut retenir. Les noms de saints ne sont pas obligatoires et la correspondance avec une trève est plutôt une exception.

32 La tref semble avoir son équivalent dans la frairie en zone romane. Cette institution fréquente, présente un caractère religieux mais relève d'une sociabilité beaucoup plus large. Souvent centrée sur une chapelle, elle étend son influence sur un quartier de paroisse. Les textes n'en parlent qu'à partir de la fin du Moyen Âge mais on peut suivre N. Y. Tonnerre quand il propose de la voir exister, au moins pour une part, à une période bien antérieure. Avessac avait douze frairies à la fin du Moyen Âge dont neuf dédiées à un saint breton mais Plélan, au XVIII ${ }^{\mathrm{e}}$ siècle, en possédait au moins trois centrées sur des clairières possédant hameau et chapelle ${ }^{50}$. Il y a bien là une 
organisation sociale dont les prémices, et sans doute plus, sont envisageables au Moyen Âge. L'attachement au sol est donc d'abord le fait de groupes restreints installés sur des espaces très inférieurs au territoire paroissial ${ }^{51}$. Le travail et même certains traits de la vie sociale semblent échapper à cette dernière entité, cela pourrait conduire à nier l'échelon paroissial et à le considérer comme une structure plaquée sans véritable existence. Une telle analyse procédant par opposition serait erronée.

Dès la fin $\mathrm{du} \mathrm{XI}$ siècle, généralement, la parrochia est devenue la référence pour situer un lieu ou une terre, elle s'inscrit comme un espace familier, connu et surtout qui sert de cadre à la vie courante. Cela signifie donc que cet espace s'est structuré, que la communauté a su se l'approprier et l'organiser de façon un peu rigoureuse, ce qui n'était pas le cas ou différemment pendant le haut Moyen Âge. Cette organisation s'exprime très concrètement par la réorganisation du réseau viaire désormais de plus en plus centré sur le pôle paroissial qui affirme ainsi sa supériorité en terme de pouvoir. Parfois, un schéma nettement rayonnant se dégage de l'étude des plans terriers ou cadastres. Des ensembles à caractère radioconcentrique se présentent, comme pour la paroisse de La Roë née d'un défrichement dirigé par l'abbaye, mais aussi dans bien des villages de la Champagne du Maine qui, à cette époque, conservait un peuplement beaucoup plus groupé. Ces cas simples se révèlent cependant assez rares. Le plus fréquent est un schéma plus complexe : le réseau viaire part du centre vers des nœuds secondaires, souvent des hameaux, qui eux-mêmes irriguent leur propre territoire. Cette maitrise du finage se complète par un balisage qui exprime aussi appropriation et contrôle. Le territoire de Livré est bordé par des cours d'eau qui sont précisément nommés mais des arbres isolés, deux grands chênes, la Croix de Triscan permettent d'identifier l'espace et de l'enserrer dans un réseau de repères.

Tout cela signifie l'accomplissement de fonctions d'intérêt général. La réclamation des habitants de Livré demandant le rattachement de la portion de forêt oubliée par le duc renvoie à des soucis très matériels. Le finage du village se présente à la fois comme terrain de culture, d'élevage et de ramassage du bois et produits divers. En ces domaines, pris dans son ensemble, il peut aussi constituer une espace de référence qui transcende celui des hameaux. Un même paysan peut avoir toutes ses terres bien groupées dans un quartier du finage villageois mais elles peuvent aussi se disperser comme à Breulis. Dans ce «village " en Noyal du Morbihan actuel, sept habitants, détenteurs d'un emplacement dans le cimetière où ils résident, ont leurs tenures disséminées sur le finage ${ }^{52}$. Bien plus, c'est l'ensemble de la communauté villageoise qui peut exercer ses droits sur les forêts et le saltus comme à Livré. Les communs peuvent aussi être liés à une paroisse. Il en est cité dans le bas-Maine pour les paroisses de Fougerolles, Oisseau, Désertines, la Tannière, toutes groupées dans l'extrémité nordouest du Maine $e^{53}$. Des procès et quelques indices laissent entrevoir un processus d'accaparement des espaces vagues par les seigneurs qui imposent progressivement des autorisations et taxes pour des usages sans doute anciens. Ces usages collectifs ne sont pas impossibles dans d'autres domaines et un texte du cartulaire de Saint-Serge d'Angers définit le pasnage des porcs à Beaupréau d'abord sur les chaumes en août puis en forêt par la suite, il y a bien une gestion commune de l'espace qui est ici paroissial ou du moins largement étendu ${ }^{54}$.

Les cadres de pouvoir n'interviennent qu'en renfort comme il a été évoqué précédemment. Ils contribuent à forger une conscience du groupe à la fois dans un système de pratiques et de taxes qui se perfectionne au cours des siècles. C'est le rôle de 
la paroisse avec la réforme pastorale du XIII ${ }^{e}$ siècle qu'accompagne la naissance des fabriques. La seigneurie, quant à elle, s'est coulée dans ce moule paroissial, elle peut même installer un ministérial responsable dans ce cadre comme le prévôt de l'abbaye Saint-Georges de Rennes installé à Pleubian ${ }^{55}$. En ce cas qui n'est pas rare, les identités se confondent et se renforcent mutuellement. Une expression villageoise peut donc naître, réunissant tous les hommes disséminés et affirmant l'emprise commune du groupe. Cette force peut puiser ses origines en des périodes fort anciennes mais on la soupçonne plus qu'on ne la connaît, le domaine du sacré se laisse pourtant un peu mieux appréhender

Une forte sacralisation cimente le lien entre le territoire et la communauté. Elle s'incarne couramment dans l'érection de croix, de nombreuses chapelles et dans la dévotion à des sources considérées comme plus ou moins miraculeuses ${ }^{56}$. Souvent, les processions y sont associées et, arpentant le territoire établissent une communion physique et sacrée. C'est sans doute aussi l'une des fonctions assumées par les Rogations souvent citées mais rarement décrites ${ }^{57}$. En Bretagne, existe un certain nombre de circumambulations autour du territoire paroissial, en premier la grande Troménie de Locronan. Tous les six ans, une longue procession de douze kilomètres suit à travers chemins et landes à peu près les limites de la paroisse en faisant douze stations et en passant devant un certain nombre de statues de saints dont certaines viennent de l'église paroissiale. Même si l'on peut évoquer un lointain sanctuaire païen et le souvenir d'un sacré préchrétien, il n'en demeure pas moins que cette procession en étroit rapport avec l'immunité d'un minihi et des donations à l'abbaye de Sainte croix de Quimperlé établit un lien très étroit entre la terre paroissiale et l'Église qui rappelle ainsi son bien mais en y associant la population qui manifeste par sa participation, attestée depuis la fin du XIII ${ }^{e}$ siècle, son attachement à son territoire ${ }^{58}$.

Plus généralement, les vies de saints reprennent souvent le thème d'un tracé par une circumambulation du fondateur. La vie de saint Lunaire raconte la délimitation d'un territoire qui deviendra celui d'une paroisse. Le son de la cloche du saint détermine par le biais de ceux qui l'entendent l'aire de ses possessions, soit un cercle de quatre milles de diamètre. Ensuite, une grande pierre, un menhir, trace miraculeusement un sillon à travers champs, landes et hameaux pour délimiter clairement cette nouvelle circonscription $^{59}$. Cette vita du XII ${ }^{\mathrm{e}}$ ou XIII ${ }^{\mathrm{e}}$ siècle est une œuvre d'ecclésiastique, bien sûr, mais B. Merdrignac a bien montré les rapports étroits entretenus entre l'hagiographe et son public et la vita rencontrait sans doute un fort assentiment populaire. Cette thématique n'est d'ailleurs pas spécifiquement celtique, on la retrouve dans le Maine pour la fondation de Saint-Calais.

À plus d'un titre, l'espace paroissial fonctionne donc bien comme un élément essentiel. Certes, il n'est pas question de voir dans le village de l'Ouest la vigueur des relations découvertes dans des zones d'habitat groupé, le modèle est différent. La référence au territoire est permanente mais se module selon les domaines. L'individu se trouve profondément engagé non pas dans un seul mais dans différents réseaux de liens qui ne s'excluent pas, bien au contraire, au moins dans le village cohérent et fortement constitué. L'individu se reconnaît membre de la communauté paroissiale dont la réalité est incontestable et qui dépasse d'ailleurs le strict domaine du religieux et du sacré. Par contre, la relation avec sa propre terre va s'exprimer plutôt dans le hameau siège d'une sociabilité basée largement sur l'entraide et le travail. 
Si l'on analyse un finage comme celui de Montourtier, en Mayenne, on perçoit très bien la juxtaposition d'espaces différents qui, au Moyen Âge, se distinguaient plus nettement en raison de leur séparation par des zones de bois ou de landes (fig. 3$)^{60}$. Le centre paroissial a son propre territoire dont l'organisation est claire. On distingue ensuite la clairière du château de Bourgon dont les anciennes coutures se reflètent dans un parcellaire encore lâche tout comme celui des prieurés de Lincé et Saint-Martin de Sézain, plus au sud. Vers l'est, l'espace est polarisé par des grosses métairies comme la Rouzière, tandis qu'à l'est un vaste ensemble circulaire voit sur son pourtour se multiplier de petits hameaux et la grange cistercienne de Champeux appartenant à l'abbaye de Fontaine-Daniel. Ces espaces ont leur vie propre mais la paroisse existe aussi et les fédère. Le modeste centre paroissial a eu assez de force pour polariser l'espace et n'a pas subi la concurrence de gros hameaux.

Fig. 3. Le finage de Montourtier (Mayenne)

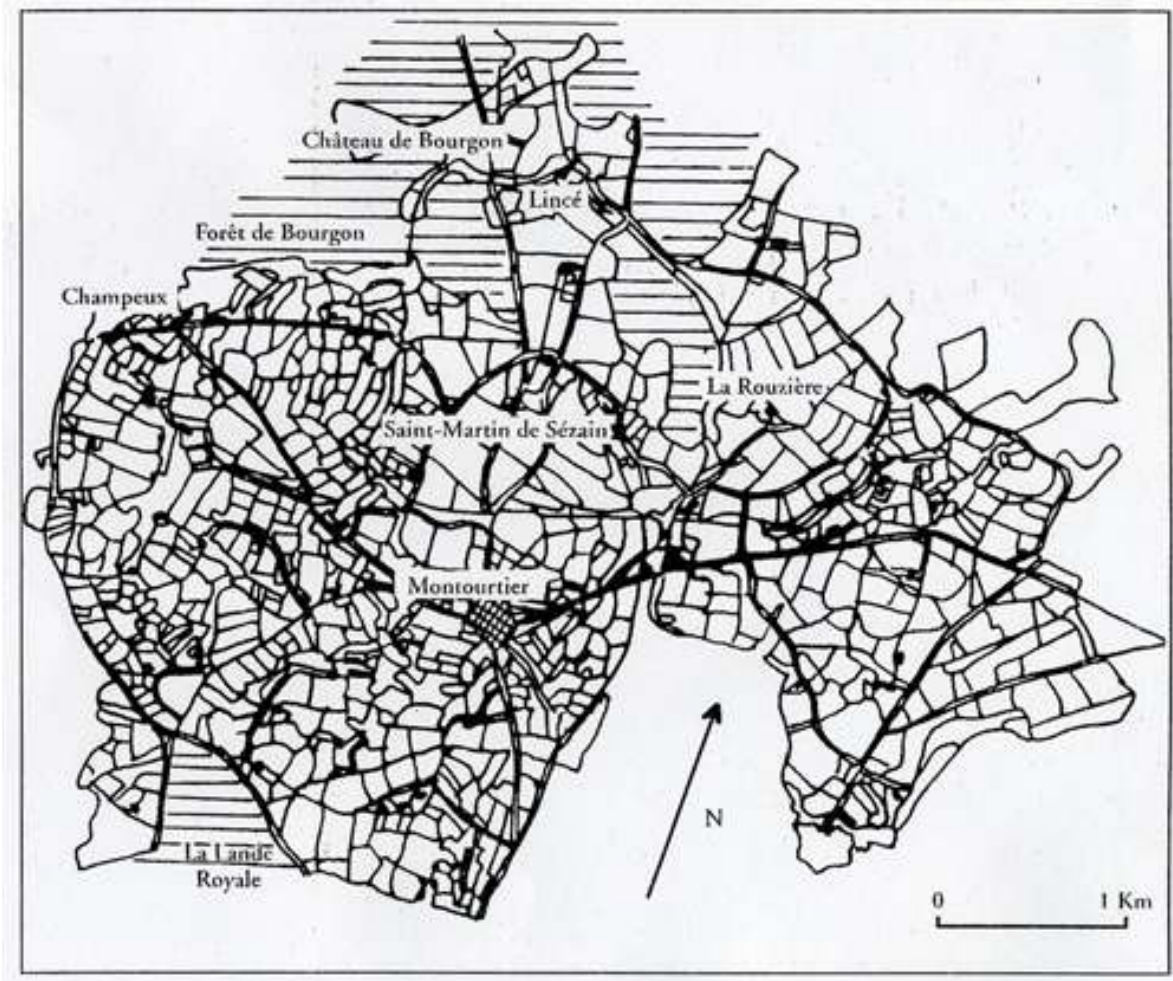

Le relevé du parcellaire a été effectué par G. Macé, Un département rural à l'Ouest : la Mayenne, Mayenne, 1982.

Cette organisation se retrouve encore dans certaines grandes paroisses primitives. Plélan semble contrôler un vaste espace où gravite une série de hameaux importants en particulier dans les clairières forestières où de forts regroupements ont leur chapelle. Elle se voit alors limitée dans ses attributions et l'on veille à lui interdire les fonctions paroissiales : baptême, sépultures etc. Certaines peuvent quand même déjà connaître le statut largement autonome de trève. La communauté de la plou demeure assez forte pour résister aux forces centrifuges et des mesures sont prises pour que le lien soit maintenu. Les paroissiens des trèves et chapelles doivent se rendre aux grandes fêtes à l'église mère, rendant ainsi concrète l'unité du groupe et du territoire qui est le sien. 
41 Si les conditions d'une vie normale ne sont pas remplies et si la force du hameau s'accroît, la rupture est consommée et une nouvelle paroisse prend son essor - c'est le cas des naissances assez nombreuses à l'époque féodale par éclatement des grandes paroisses. Les paroisses primitives, les plous bretonnes très vastes n'ont pas toujours, aux hautes époques, une délimitation bien précise mais le fait que beaucoup ne seront pas démembrées à la période féodale malgré la croissance démographique est révélateur d'une fidélité territoriale. On préfère le système des trèves qui maintient des quasi-paroisses dans un cadre plus large. S'il est assez logique d'invoquer la volonté épiscopale, il est cependant à envisager fortement le souhait des paroissiens qui ne voulaient pas toujours scinder la communauté et son territoire.

La cohésion et l'organisation évoquées trouvent leurs limites et l'espace des paroissiens peut être plus éclaté. Les centres faibles ou dédoublés peinent sans doute à maintenir cette unité et Belgeard, formé de la réunion de cinq gros hameaux de défrichement pratiquement équivalents, manque singulièrement d'unité et l'on peut s'interroger pour les églises et cimetières qui n'ont pu provoquer l'agglomération d'un minimum d'habitants à leurs abords ${ }^{61}$. Certaines, limites demeurent suffisamment indécises pour que des sites soient par alternance d'une paroisse ou d'une autre comme les tournes du Maine connues à l'époque Moderne. D'ailleurs, le paysan peut dépasser ce cadre, posséder des terres en dehors de la paroisse et les espaces de parcours peuvent eux aussi s'étendre sur le voisinage. Les vastes landes des Bercons étendues sur cinq paroisses près de Ségrie dans la Sarthe actuelle attirent les habitants des alentours ${ }^{62}$. L'espace paroissial s'emboîte dans d'autres espaces tout en restant un cadre d'organisation dominant.

L'espace est bien, dans l'Ouest, l'un des paramètres majeurs de la communauté villageoise. Il se définit vraiment au moment où s'organise le village dans ses formes nouvelles, c'est-à-dire quand le peuplement s'établit globalement autour des châteaux mais surtout des centres paroissiaux. S'il peut être neuf, cet espace est aussi héritier des temps anciens. Son découpage ou sa réorganisation sont certes liés aux autorités qui encadrent les hommes et contrôlent donc l'espace mais ces dernières ne peuvent le faire qu'en tenant compte largement des liens établis avec les communautés déjà existantes ou qui s'esquissent. Cet espace se charge donc d'une foule de références.

La relation s'établit cependant à différents niveaux et il faut reconnaître, en particulier, un échelon extrêmement fort, celui du hameau qui s'insère comme un relais et assume un rôle essentiel assez différent de celui du centre. Sauf cas de concurrence qui peut miner la communauté, l'ensemble s'organise suivant de subtils équilibres complémentaires. Les hommes s'intègrent en fait dans différents espaces aux échelles différentes, suivant le type de référence en jeu. Cela implique l'intégration dans des communautés réelles et vivantes mais pas antinomiques. Un tissu complexe de relations sociales, de solidarités et de conflits s'inscrit dans ces territoires qui ont une existence incontestable. Cette vigueur explique sans doute leur pérennité. Fixés souvent avant le XIII ${ }^{\mathrm{e}}$ siècle, ils n'ont guère bougé depuis, même si les paroisses sont devenues communes. Le découpage opéré à l'époque féodale ne définit pas un simple maillage mais détermine des espaces que les hommes ont organisés en de véritables territoires fondateurs de leur identité. 


\section{NOTES}

1.D. Pichot, Le village éclaté. Habitat et société dans les campagnes de l'Ouest au Moyen Âge, Rennes, PUR, 2002, $395 \mathrm{p}$.

2.On ne peut que reprendre la définition du village proposée par R. Fossier, Enfance de l'Europe, aspects économiques et sociaux, Paris, PUF, 1982, pp. 191-193.

3.D. Pichot, op. cit., chap. 5.

4.R. Brunet, Géographie universelle, t. 1, Mondes nouveaux, p. 35, voir aussi, M. Le Berre, "Territoires », A. Bailly, R. Ferras, D. Pumain, Encyclopédie de la Géographie, Economica, 1995.

5.B. Merdrignac, Les vies de saints bretons durant le haut Moyen Âge, Rennes, 1993, pp. 123-128, D. Laurent, « Le juste milieu. Réflexion sur un rituel de circumambulation millénaire : la troménie de Locronan ", Tradition et histoire dans la culture populaire. Rencontres autour de l'œuvre de J. M. Guilcher, Documents d'Ethnologie Régionale, vol. XI, Centre Alpin et Rhodanien d'ethnologie, Grenoble, 1990.

6.E. Lorans, Le Lochois du haut Moyen-Âge au XIII siècle. Territoires, habitats et paysages, Tours, 1996, pp. 48-49.

7.G. Louise, La seigneurie de Bellême Xe-XIIe siècles. Le Pays Bas-Normand, 1993, t. 2, p. 83.

8. On peut citer les vici de Saulges ou d'Entrammes dans la Mayenne, globalement, dans le Maine on en compte au moins 70 dont la plupart ont donné naissance à des communes modernes.

9.A. Bertrand de Broussillon éd., Cartulaire de l'abbaye Saint-Aubin d'Angers, Angers, 1903, $\mathrm{n}^{\circ} 85$.

10.Y. Hillion, Le chartrier de l'abbaye Notre Dame d'Evron (essai de reconstitution), Thèse de troisième cycle, Rennes, 1977, T. II, vol. 1, p. 19, Evron, com. et c., Mayenne, les villages cités sont à proximité.

11.Sur les plou, la bibliographie est importante, on peut se référer à N. Y. Tonnerre, Naissance de la Bretagne, géographie historique et structures sociales de la Bretagne méridionale (Nantais et Vannetais) de la fin du VIII è la fin du XII e siècle, Angers, 1994, 626 p.

12.M. Planiol, « La donation d'Anouuareth », Annales de Bretagne, t. 9, 1893-1894, pp. 237-294.

13.E. Zadora-Rio, "L'église et le regroupement de l'habitat en Anjou aux $\mathrm{XI}^{\mathrm{e}}$ et $\mathrm{XII}{ }^{\mathrm{e}} \mathrm{s}$. ", M. Fixot et E. Zadora-Rio, dir., L'environnement des églises et la topographie religieuse des campagnes médiévales, DAF, 1994, pp. 137-148.

14.A. Debord, « L'église, le château et l'organisation de l'espace dans l'ouest de la France, $\mathrm{X}^{\mathrm{e}}$-XIV ${ }^{\mathrm{e}}$ siècles ", A. Chastel dir., Cahiers de Commarque, $n^{\circ} 1$, L'Église et le château, p. 36.

15. E. Zadora-Rio, Rigny-Ussé, Archéologie d'une commune rurale, Conseil régional de la région Centre, 1992, $38 \mathrm{p}$.

16.M. Le Mené, Les campagnes angevines à la fin du Moyen Âge, 1350-1530, étude économique, Nantes, 1982, p. 126.

17.Arch. dép. Mayenne, Cartulaire de La Roë, E. Laurain éd., "Questions fabriciennes », Bulletin de la Commission historique et archéologique de la Mayenne, t. 24, 1908, pp. 348-349. 18.J. M. Bienvenu, « Un défrichement angevin au temps de la réforme grégorienne : la co-seigneurie de Belle-Noue ", Annali d'historia economica e sociale, 8, 1967, pp. 75-90.

19.D. Pichot, Le village éclaté, op. cit., p. 299. 
20." Antequam episcopus benedicere cimiterium inciperet, inquisivit a domino R[eginaldo] Allobroge utrum essent aliqui habitantes circa nos in foresta qui possent libere esse parrochiani hujus cimiterii quod benedicere volebat ", Cartulaire de La Roë, 3, ibidem.

21.D. Pichot, Le village éclaté, op. cit., p. 133.

22.A. Chédeville, « Un défrichement en Bretagne à la fin du XI ${ }^{\mathrm{e}}$ siècle : histoire économique et géographie féodale », J.-M. Duvosquel and E. Thoen, Peasants and townsmen in medieval Europe. Studia in honorem Adriaan Verhulst, Centre belge d'histoire rurale, $\mathrm{n}^{\circ}$ 114, Gand, 1995, pp. 429-444, La Lande-Huan, com. Lanrigan, c. Combourg, Ille-et-Vilaine.

23.D. Pichot, Le village éclaté, op. cit., p. 182, 145, La Roë, c. Saint-Aignan-sur-Roë, Mayenne ; Vautorte, c. Ernée, Mayenne ; Brigne, com. Bousse, c. Malicorne, Sarthe. 24.E. Zadora-Rio, « Rigny », op. cit.

25.Gahard, c. St-Aubin-d'Aubigné, Ille-et-Vilaine. H. Guillotel, Les Actes inédits des ducs de Bretagne (944-1148), thèse, Université de Paris 2, 1973, ms dactyl., nº 19 (1008-1031).

26.Idem, "Terminabatur autem idem locus terra sancti Albini, terra sancti Germani Veteris vici et sancti Martini de ecclesiaa vici qui Macerias dicitur cum via publica».

27.A. de La Borderie, Recueil d'actes inédits des ducs et princes de Bretagne (XI ${ }^{e}-X V^{e}$ siècles), Paris, 1899, n² 2, 11-23, H. Guillotel, Actes, op. cit., 62, 96, Livré, c. Liffré, Ille-et-Vilaine.

28. Ergo Livriacus dicitur ipsa elemosyna quae sic divisa est comitis Gauzfridi jussu : ab ecclesia usque ad terram que nuncupatur Vendeliscum, sicut Veouris currit usque ad torrentem Birzillae, atque de torrente ad publicam viam Redonensem uti dirigitur ad Rachatam perquercum Pediculosum quercumque Furcatum ", H. Guillotel, nº 62.

29." [...] quamdam partem sylvaticae terrae adhaerentem Livriacensi parrochiae quam Livriacenses antiqui affirmabant quondam fuisse Sancti Florentiii, cum caetera terra Livriacensi. Protenditur autem praedicta pars ab aïa Ingelsendis usque ad Burgundinariam per crucem Triscandi », ibidem, H. Guillotel, $n^{\circ} 96$.

30.Fr. Hervé, La forêt de la Hunaudaye et ses abords. Esquisse de l'évolution des paysages, Mémoire de maîtrise, Rennes 2, 1991, pp.87-100. La forêt se trouve dans l'est des Côtes d'Armor.

31.D. Pichot, Le village éclaté, op. cit. p. 341.

32.Arch. dép. Mayenne, ms 32, n 105, Landivy, com. et c., ar. Mayenne.

33.A. Chédeville et N. Y. Tonnerre, La Bretagne féodale (XIe-XIIIe siècle), Rennes, 1987, pp. 301-307.

34.M. Planiol, La très ancienne coutume de Bretagne avec les Assises, Constitutions de parlement et Ordonnances ducales, Paris-Genève, 1984, art.39, 219, M. C. BeautempsBeaupré, Coutumes et institutions de l'Anjou et du Maine antérieures au XVI siècle, Paris, t. 1, 1877, p. 133.

35.P. Flatrès, « La structure rurale du Sud-Finistère d'après les anciens cadastres ", Norois, $\mathrm{n}^{\circ} 15,1957$, pp. 353-367, $\mathrm{n}^{\circ} 16$, pp. 425-453; « L'étendue des finages villageois en Bretagne », Norois, t. 18, 1968, pp. 181-189.

36.G. Astill et W. Davies, A breton landscape, Cambridge, 1998, chap. 6.

37.A. Meynier, Les paysages agraires, Paris, 1958, pp. 30-32.

38.J. Renard, Les évolutions contemporaines de la vie rurale dans la région nantaise, LoireAtlantique, Bocages vendéens, Mauges, Les Sables d'Olonne, 1976.

39.J. F. Duval, Occupation et mise en valeur du sol dans la région de Plélan-le-Grand au Moyen Âge, Mémoire de maîtrise, Rennes 2, 1998.

40.J. R. Trochet, Aux origines de la France rurale. Outils, pays et paysage. Mémoires et documents de Géographie, CNRS, 1993, pp. 16-17. 
41.P. André, « Un village médiéval breton du XI ${ }^{\mathrm{e}}$ siècle : Lann-Gouh en Melrand (Morbihan) ", Archéologie médiévale, t.12, 1982, pp. 155-174.

42.D. Pichot, op. cit., pp. 189-190.

43.Ibidem, p. 338.

44.S. Reynolds, Kingdoms and Communities in Western Europe 900-1300, 2e ed., Oxford, 1997, p. 113.

45.Arch. dép. Mayenne, $\mathrm{ms} 33, \mathrm{n}^{\circ} 62$.

46.M. Le Mené, « La forêt du Lattay au Moyen Âge. Défrichement de sa partie occidentale entre la Loire, l'Evre et Hyrome », Le Moyen Âge, t. 76, 1970, pp. 27-60.

47.B. Tanguy, « De la treb à la trève ou de l'origine des frairies et des trèves ", Chrétientés de Basse-Bretagne et d'ailleurs, Mélanges offerts au chanoine Jean-Louis Le Floc'h, Société archéologique du Finistère, 1998, pp. 237-244.

48.Ibidem. Pleubian, com et c. Lezardrieux, ar. Lannion, Côtes d'Armor. L

49.D. Pichot, op. cit., pp. 223-224.

50.N. Y. Tonnerre, op. cit., pp. 454-457, Chanoine Guillotin de Corson, Pouillé historique de l'archevêché de Rennes, Paris-Rennes, 1880-1886, art. Plélan, paroisse.

51.Cela rejoint les travaux de $\mathrm{B}$. Derouet qui à l'époque moderne relève le fort attachement de groupes restreints à leur territoire, B. Derouet, « Territoire et parenté. Pour une mise en perspective de la communauté rurale et des formes de reproduction familiales ", AHSS, mai-juin 1995, pp. 645-686.

52.A. de Courson, Le cartulaire de Redon, $\mathrm{n}^{\circ} 375$, Noyal, c. Muzillac, ar. Vannes, Morbihan. 53.D. Pichot, Le Bas-Maine du Xe au XIII ${ }^{e}$ s., étude d'une société, Laval, 1995, pp. 268-269.

54." [...] pasnaticum porcorum post messionem segetum in stipulis in augusto, et post augustum pasnaticum bosci... ", Cartulaires de Saint-Serge..., t. 2, 1, 66, p. 335.

55.P. de La BigneVilleneuve, Cartulaire de l'abbaye Saint-Georges de Rennes, Rennes, 1876, $\mathrm{n}^{\circ} 19$.

56.C. Prigent, « Un exemple de survivance de traditions anciennes en Basse-Bretagne : les fontaines thérapeutiques ", Chrétientés de Basse-Bretagne et d'ailleurs, op. cit., pp. 153-159.

57.Les Rogations sont connues dès le $\mathrm{XI}^{\mathrm{e}}$ siècle, elles figurent souvent dans les actes de restitution d'églises.

58.B. Merdrignac, Les vies de saints bretons, op. cit., pp. 123-128.

59.23 « Post hec rogavit sanctus Leonorius sicut Gillebertus rex illi commendavit cimbalum super unius montis cacumen sonare suum ubicumque sonitus eius fuisset auditus terra illa per donationem regis usque in finem seculi sua fuisset. Vox namque cimbali, Deo juvante atque volente, in tantum fuit vociferata ut per quatuor miliaria in circuitu equali modo ex omni parte fuit audita [...] Et hec dicens elevavit se a terra et cum eo similiter lapis erexit se. Tunc sanctus Leonorius tunicam accepit suam et jactavit eam super cacumen lapidis et imperavit illi sicut ab angelo Domini monitus est ut supradiximus et statim lapis antecedit eum et cunctum populum sequentem, gradiens et per planam terram et per montes altos et per valles humiles necnon et per villas, domus frangens, quousque ad illum de quo perrexit locum arans et sulcans terram pervenit. ", A. Carrée et B. Merdrignac, La vie latine de saint Lunaire, textes, traduction, commentaires, Britannia monastica, Landevennec, 1991, pp. 154-156.

60.Montourtier, c. Montsûrs, ar. Laval, Mayenne. D. Pichot, Le village éclaté, pp. 281-283. 61.Belgeard, c. et ar. Mayenne, Mayenne.

62.A. Bouton, Le Maine, histoire économique et sociale, le Moyen Âge, Le Mans, 1976, pp. 266-267. 


\section{AUTEUR}

\section{DANIEL PICHOT}

Université Rennes-II, Centre de recherches historiques sur les sociétés et cultures de l'Ouest européen (CRHISCO) 